This item was submitted to Loughborough's Research Repository by the author.

Items in Figshare are protected by copyright, with all rights reserved, unless otherwise indicated.

\title{
Laser vibrometers and contacting transducers, target rotation and six degree-of-freedom vibration: what do we really measure?
}

PLEASE CITE THE PUBLISHED VERSION

http://dx.doi.org/10.1006/jsvi.2000.3053

\section{PUBLISHER}

Elsevier () Academic Press

VERSION

AM (Accepted Manuscript)

\section{LICENCE}

CC BY-NC-ND 4.0

\section{REPOSITORY RECORD}

Bell, John R., and Steve Rothberg. 2019. "Laser Vibrometers and Contacting Transducers, Target Rotation and Six Degree-of-freedom Vibration: What Do We Really Measure?”. figshare.

https://hdl.handle.net/2134/9663. 
This item was submitted to Loughborough's Institutional Repository (https://dspace.lboro.ac.uk/) by the author and is made available under the following Creative Commons Licence conditions.

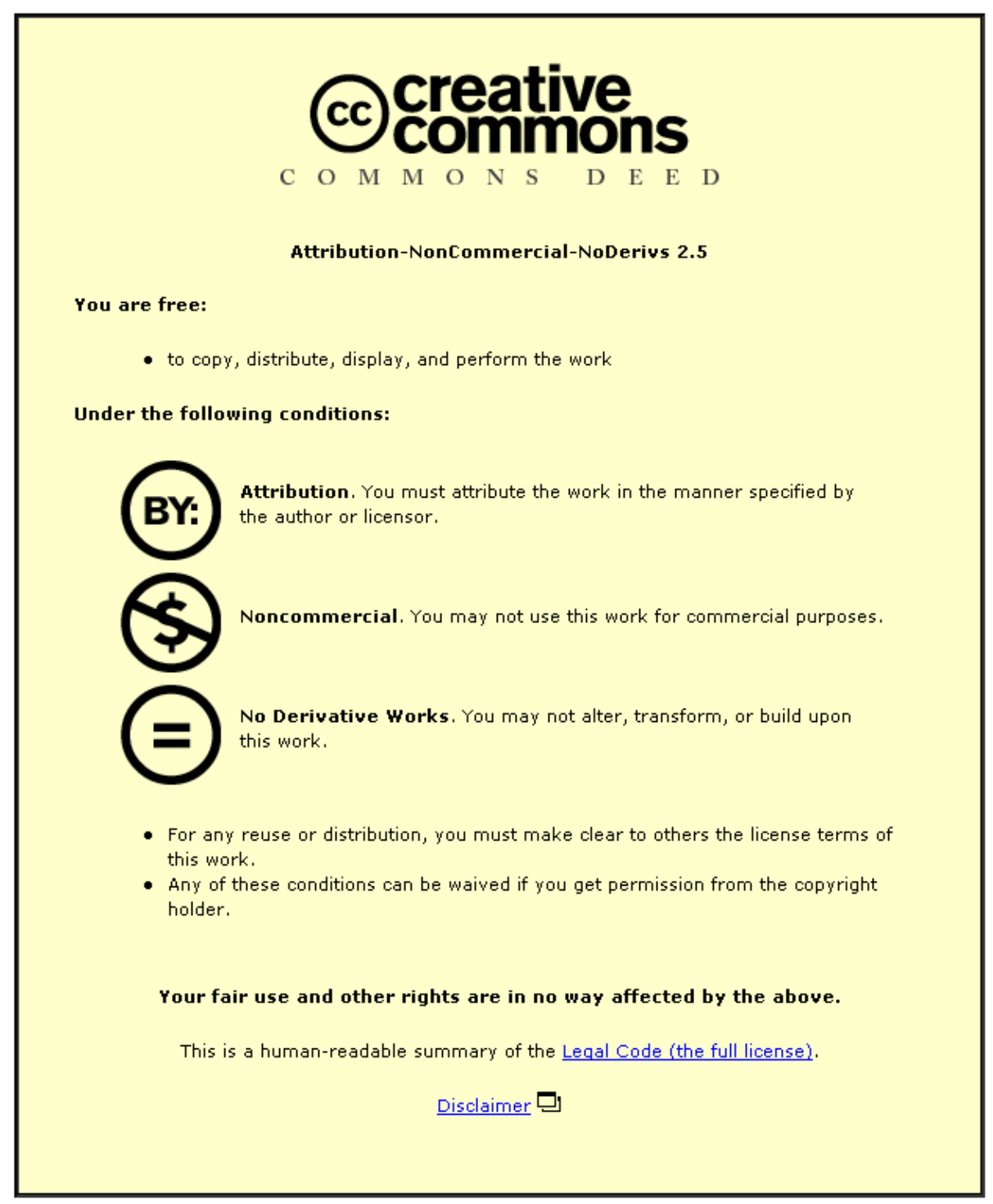

For the full text of this licence, please go to: http://creativecommons.org/licenses/by-nc-nd/2.5/ 
LASER VIBROMETERS AND CONTACTING TRANSDUCERS,

TARGET ROTATION AND 6 DEGREE-OF-FREEDOM VIBRATION:

WHAT DO WE REALLY MEASURE?

\author{
J. R. BELL and S. J. ROTHBERG \\ Department of Mechanical Engineering, Loughborough University \\ Loughborough, Leicestershire, UK
}

Total Number of Main Text Pages: 25

Total Number of Figures: 7 in 10 pieces

Short Title: LASER VIBROMETERS AND 6 DEGREE OF FREEDOM VIBRATION

\title{
Address for Correspondence:
}

Dr S. J. Rothberg

Department of Mechanical Engineering

Loughborough University

LOUGHBOROUGH

Leicestershire LE11 3TU 


\section{SUMMARY}

This paper presents a comprehensive analysis of the velocity sensed by a single laser vibrometer beam incident in an arbitrary direction on a target that is of substantial interest in engineering - a rotating shaft requiring three translational and three rotational co-ordinates to describe its vibratory motion fully. Six separate "vibration sets", each a combination of motion parameters, appear in the full expression for vibration velocity sensitivity and it is shown not to be possible to resolve individual motion components within each set by arrangement, or even manipulation, of laser beams. To place this non-contact transducer velocity sensitivity model in its proper context, the velocity sensitivity of a contacting transducer under identical conditions is also derived and comparison is made between noncontacting and contacting transducer performance. Specific applications of the laser vibrometer theory to radial and axial vibration measurements are set out and it is shown how estimation of radial vibration components is only possible by post-processing. The theory is easily extended to include measurements made with multiple beams, underlining the model's versatility in enabling determination of the vibration component sensitivity of a measurement with any beam orientation or combination of beam orientations. This will prove beneficial in devising further optical configurations for measurement of torsional, pitch and yaw vibrations. 


\section{INTRODUCTION}

The principle of Laser Doppler Vibrometry (LDV) relies on the detection of the Doppler frequency shift in coherent light scattered from a moving target. By measuring the frequency shift, a time resolved measurement of the target velocity is made. The non-contact nature of Laser Vibrometers offers significant advantages over traditional contacting vibration transducers and measurements on hot, light or rotating components are often cited as important applications.

For rotors, vibration measurement is important from the earliest stages of design and development through to condition monitoring of installed machinery. Measurement of the vibration transmitted from the rotor into a non-rotating component is the most common option but, for example, where the rotor is mounted into large or rigid bearings, low vibration transmission can make this unreliable. In many cases, a non-contact vibration transducer capable of measuring directly from any location along the rotor is desirable and LDV offers this possibility. Indeed, one of the first reported LDV applications was for vibration measurement directly from a rotating turbine blade [1]. Investigations of magnetic discs [2,3], bladed discs [4,5] and modal analysis on rotating discs [6] are typical and more recent examples of measurements that can be made.

LDV measurements made on rotors, however, can be ambiguous, affected by vibration components perpendicular to the component it is intended to measure. A feature of much previous work has been prediction of acceptable performance in the presence of a single vibration component, neglecting the effects of other components present in the more complex motions likely to be encountered in practice. When previous studies have acknowledged cross-sensitivities, notably to radial vibration measurements [7] and torsional vibration measurements [8], these have merely been special cases of the totally general theory to be presented in this paper. Interestingly, investigators have already reported designs for a three 
dimensional vibrometer [9] and a six-degree-of-freedom vibrometer [10]. Until now, however, there has been no analysis of the velocity sensed by a single laser beam incident in an arbitrary direction on a target that is of substantial interest in engineering - a rotating shaft requiring three translational and three rotational co-ordinates to describe its vibratory motion fully. This new theory is placed in its proper context by a similarly comprehensive description of the velocity measured under the same conditions by a contacting transducer.

The theory will be a useful tool for the engineer, allowing the sensitivity of any measurement to be predicted easily for any combination of target motion components. In addition, remaining fundamental questions about the use of laser vibrometers on rotating components will finally be answered.

\section{TOTAL VELOCITY MEASURED BY A NON-CONTACT LASER VIBROMETER}

\subsection{VELOCITY AT THE POINT OF INCIDENCE OF THE LASER BEAM}

The case considered is that of a rotating shaft, of arbitrary shape, undergoing an arbitrary vibration requiring three translational and three rotational co-ordinates for description. The resulting theory, however, will be equally applicable to any non-rotating, vibrating structure. As shown in Figure 1, a translating reference frame $x y z$, which maintains its direction at all times, has its origin $\mathrm{O}$ fixed to a point along the spin axis within the shaft. $\mathrm{P}$ is the instantaneous point of incidence of the laser beam on the shaft and is identified by the position vector $\vec{r}_{P}$. Provided that the illuminated axial element of the shaft can be assumed to be rigid, the velocity of P, $\vec{V}_{P}$, is the sum of the translational velocity of origin $\mathrm{O}, \vec{V}_{0}$, and the velocity of $\mathrm{P}$ relative to $\mathrm{O}$ as a result of rotation about an instantaneous rotation axis passing through $\mathrm{O}$ at angular velocity $\vec{\omega}$ : 


$$
\vec{V}_{P}=\vec{V}_{o}+\left(\vec{\omega} \times \vec{r}_{P}\right)
$$

where $\vec{V}_{0}=V_{x} \hat{x}+V_{y} \hat{y}+V_{z} \hat{z}$ and $\vec{\omega}=\dot{\theta}_{x} \hat{x}+\dot{\theta}_{y} \hat{y}+\dot{\theta}_{z} \hat{z}+\Omega \hat{z}_{R}$. Note how the unit vector defining the rotation axis, $\hat{z}_{R}$, differs from the unit vector $\hat{z}$ which is defined by the undeflected shaft rotation axis.

In the usual configuration, a laser vibrometer measures target velocity at the point of incidence in the direction of the probe laser beam. The position vector $\vec{r}_{P}$ can be used to define the point in space where the line of the laser beam intersects the surface of the shaft. However, as the shaft vibrates and rotates, the position of this point, not only on the target but also in space, will change continuously, becoming a function of time.

Figure 2 shows three ways in which the position in space of the incident point can vary from an initial location, shown by a ' $X$ ' in each figure, to a final location, shown by a ' $\bullet$ '; firstly due to translation of the shaft, secondly due to variations in the shape of the shaft as it rotates (or rolls) and finally due to pitch and yaw of the shaft. (Note that "shape variation", as used in this paper, refers to variations in shaft dimensions and not to any dynamic shape variation). Since the shaft can have an arbitrary shape and it would be inconvenient to have the velocity measured described in terms of shape, this would appear to be a difficulty in progressing this analysis.

A reliable piece of information, however, is that, no matter how the shaft moves, the point of incidence will always lie somewhere along the line of the beam. Any point on the line of the beam can be described as the sum of the position vector of a known point $\vec{r}_{0}$ that lies on the line of the beam, $\hat{b}$, and a multiple of the unit vector defining the direction of the beam. At some time, $t$, the position of the point of incidence $\vec{r}_{P}(t)$ will have changed from the initial position due to shaft motion and/or shape, as outlined previously, and the new point of 
incidence is depicted in Figure 3. The shaft has undergone the translation $\vec{A}(t)$ and the crosssection on which the beam is now incident has also changed due to shaft motion and/or shape. The new position of the incident point can be written as:

$\vec{r}_{P}(t)=\left[\vec{r}_{0}-\vec{A}(t)\right]+p(t) \hat{b}$

$p(t)$ is always unknown but it will prove to be a convenient quantity to account for changes in the point of incidence. This new approach simplifies the analysis required considerably compared to previous studies.

The velocity measured by the laser vibrometer, $U_{m}$, is the component of the velocity of the changing incident point in the direction of the incident beam:

$U_{m}=\hat{b} \cdot \vec{V}_{P}=\hat{b} \cdot \vec{V}_{0}+\hat{b} \cdot\left(\vec{\omega} \times\left[\vec{r}_{0}-\vec{A}\right]\right)+\hat{b} \cdot(\vec{\omega} \times p \hat{b})$

The second scalar triple product in equation (3) is always zero as can be seen more clearly by a simple re-arrangement:

$\hat{b} \cdot(\vec{\omega} \times p \hat{b})=p \vec{\omega} \cdot(\hat{b} \times \hat{b})$

This important result means that the measured velocity, $U_{m}$, is independent of the unknown parameter $p$, the parameter used to account for changes in space of the point of incidence due to shaft motion and shape variation. This proves, more generally than in any previous study, that the velocity measured by a laser vibrometer incident on a vibrating shaft is insensitive to the shape of the shaft, despite the fact that the incident beam can change axial and radial 
position on the shaft in any arbitrary fashion. Such immunity to target shape gives this measurement technique a significant advantage over, for example, proximity probe measurements. Of course the same shape immunity is found for measurements on targets undergoing simpler motions and the analysis still holds for scanning applications where $\hat{b}$ is a function of time.

\subsection{VELOCITY MEASURED BY A LASER BEAM INCIDENT ON A ROTATING}

\section{SHAFT}

Using the general theory presented above, the velocity measured by a laser beam incident on an axial element of shaft, of arbitrary shape, rotating about its spin axis whilst undergoing an arbitrary vibration can be derived. This derivation is detailed in section 1 of Appendix 1 where the substitutions $\vec{A}=a_{x} \hat{x}+a_{y} \hat{y}+a_{z} \hat{z}$ and $\vec{r}_{0}=x_{0} \hat{x}+y_{0} \hat{y}+z_{0} \hat{z}$ are used. Although $\vec{r}_{0}$ can be any point along the line of the beam, in practice the initial illuminated point is often chosen. The velocity measured by a laser beam, orientated according to the angles $\alpha$ and $\beta$ (refer to Figure 4 ) and incident on a rotating shaft is given by:

$$
\begin{aligned}
U_{m} & =\cos \beta \cos \alpha\left[V_{x}+\left(\dot{\theta}_{z}+\Omega\right) a_{y}-\left(\dot{\theta}_{y}-\Omega \theta_{x}\right) a_{z}\right] \\
& +\cos \beta \sin \alpha\left[V_{y}-\left(\dot{\theta}_{z}+\Omega\right) a_{x}+\left(\dot{\theta}_{x}+\Omega \theta_{y}\right) a_{z}\right] \\
& -\sin \beta\left[V_{z}-\left(\dot{\theta}_{x}+\Omega \theta_{y}\right) a_{y}+\left(\dot{\theta}_{y}-\Omega \theta_{x}\right) a_{x}\right] \\
& -\left(y_{0} \sin \beta+z_{0} \cos \beta \sin \alpha\right)\left[\dot{\theta}_{x}+\Omega \theta_{y}\right] \\
& +\left(z_{0} \cos \beta \cos \alpha+x_{0} \sin \beta\right)\left[\dot{\theta}_{y}-\Omega \theta_{x}\right] \\
& +\left(x_{0} \cos \beta \sin \alpha-y_{0} \cos \beta \cos \alpha\right)\left[\dot{\theta}_{z}+\Omega\right]
\end{aligned}
$$

Derivation of equation (5) is a significant step forward in the use of non-contacting transducers, allowing the vibration engineer to be sure of vibration component sensitivity for 
any laser beam arrangement on any target, including a rotating shaft. It shows that the measured velocity is the sum of six terms, each the product of a combination of geometric parameters and a combination of motion parameters - the "vibration sets". The six "vibration sets”, shown in square brackets, are inseparable combinations of different motion parameters. This important result shows that, no matter how a laser beam is aligned, only the combinations of motion parameters within the square brackets can be measured directly.

The first description of the cross-sensitivity of radial vibration measurements using laser vibrometers [11] demonstrated how the "error terms" in the measured velocity, (principally $\left(\dot{\theta}_{z}+\Omega\right) a_{y}$ or $\left.\left(\dot{\theta}_{z}+\Omega\right) a_{x}\right)$ in equation (5), could be of sufficient magnitude to mask the intended measurements of the radial velocity, $\vec{V}_{x}$ or $\vec{V}_{y}$. A particular problem in the measurement of synchronous radial vibrations was also highlighted. Since this first description, there has been discussion about whether a particular arrangement of laser beams or a particular variation of the arrangement, for example by scanning the laser beams, might enable resolution of individual motion components. Equation (5) shows that this is not possible.

The task is simplified enormously on a non-rotating target $(\Omega=0)$ but, for a rotating shaft undergoing an arbitrary vibration, direct measurement of pure radial, axial or bending vibration is not possible because the measurement will always be sensitive to other motion components. It may be possible to assume the effects of additional shaft motions are negligible, enabling direct measurement. For example, if the amplitudes of the vibration components are known, somehow, to be similar then the intended measurement dominates at vibration frequencies much higher than rotation frequency. In a general case, however, reliable estimation of components requires post-processing [12]. In contrast, unambiguous 
measurement of the axial element's time-resolved rotation speed appears possible, accepting that the torsional vibration and roll motion of the shaft are indistinguishable.

\subsection{ISOLATING INDIVIDUAL COMPONENTS}

Throughout the remainder of the discussion, the six vibration sets in equation (5) will be referred to by the vibration parameter in each group that might be regarded as the intended measurement. These are, in the order that they are presented in equation (5): the $x$ radial, $y$ radial, axial, pitch and yaw vibration sets and the rotation speed set which includes torsional vibration. Equation (5) can be simplified by setting $z_{0}=0$ so that the plane of the origin of the $x y z$ axes and the "measurement plane" are coincident, since this is just a matter of definition.

Isolation of any one of the six sets requires appropriate choice of values for $\alpha, \beta, x_{0}$ and $y_{0}$. A radial and an axial vibration measurement are shown below as examples. While the pitch and yaw vibration sets and the rotation speed set can be eliminated from a measurement, no values exist that can isolate these sets. Isolation of any of these sets requires the geometric coefficients of the three translational vibration sets to equal zero i.e. $\cos \beta \cos \alpha=\cos \beta \sin \alpha=\sin \beta=0$, to which there is no solution. Measurements made with a single laser beam will always be sensitive, therefore, to either radial or axial vibration or both, while the rotational vibration sets require more than one laser beam to be isolated.

\section{Radial vibration measurement}

To measure the $x$ radial vibration set requires alignment of the laser beam so that it passes through the centre of the shaft and along the $x$-axis making $\alpha=\beta=0^{\circ}$ and $y_{0}=0$. The measured velocity is then equal to: 


$$
U_{m}=\left[V_{x}+\left(\dot{\theta}_{z}+\Omega\right) a_{y}-\left(\dot{\theta}_{y}-\Omega \theta_{x}\right) a_{z}\right]
$$

Similarly, $\alpha=90^{\circ}, \beta=0^{\circ}$ and $x_{0}=0$ enable the $y$ radial vibration set to be isolated. Equation (6) shows agreement with previous two dimensional theory for radial vibration measurements [7] which was validated experimentally over a range of vibration amplitudes, frequencies and shaft rotation speeds, including speed fluctuations. This equation, however, extends the theory to include motion of the shaft in all six degrees-of-freedom. In particular, equation (6) reveals a third and previously unreported term in the measured velocity, $\left(\dot{\theta}_{y}-\Omega \theta_{x}\right) a_{z}$. Attempts to resolve $x$ and $y$ radial motions by post-processing [12] currently rely on the assumption that this third term is an order of magnitude smaller than the first two. Radial measurements made during experimental studies on rotating turbine blades $[1,4]$ have shown satisfactory results because only single vibration components were present. In practice, rotating structures may have motion in all six degrees-of-freedom and the measurement can therefore be ambiguous with the cross-sensitivity significant enough to mask the intended measurement. In addition to the terms in equation (6), measurements on rotating blades generally have the laser beam offset from the centre of the shaft $\left(y_{0} \neq 0\right)$ so the measured velocity will contain terms from the rotation speed set.

Experimental validation of the previously unreported terms in equation (6), $\dot{\theta}_{y} a_{z}$ and $\Omega \theta_{x} a_{z}$, was carried out using the test rig shown in figure 5 which allowed simultaneous axial and angular vibration of a small test rotor. Selection of each term was achieved by orientation of the incident laser beam first in a vertical radial direction then in a horizontal radial direction. The combination of axial and angular vibrations creates sum and difference frequency components in the measured velocity. In the validation, the motions were driven at different frequencies to distinguish the appropriate components, which are small in magnitude, from 
other vibrations produced by the mechanism used to generate the required motion of the rig. The driving frequencies were carefully chosen so that the sum and difference components were distinct and did not coincide with harmonics of either of the driving frequencies.

Figures $6 a \& b$ show the measured sum and difference frequency velocity amplitudes for a variety of vibration amplitudes along with the "theoretical" values derived from measurements using piezoelectric accelerometers fixed to the bearing housing of the shaft. Measurements of angular vibration were obtained by subtracting the outputs of two accelerometers separated by a known distance with the axial measurements made in the usual way. Combinations of two rotation speeds (nominally $28 \mathrm{~Hz}$ and $48 \mathrm{~Hz}$ ), three axial vibration amplitudes (nominally 50, 250 and 500 $\mu \mathrm{m}$ ) and three angular vibration amplitudes (nominally 30, 150 and 300mrad/s for $\dot{\theta}_{y}$ and 1.5, 7.5 and $15 \mathrm{mrad}$ for $\theta_{x}$ ) were used and the data sets numbered in ascending magnitude of the theoretical component for convenience. The measured data show reasonable agreement with the theoretical data with the differences attributed, at least in part, to genuine differences between the motion of the point probed by the laser and the points at which accelerometers were located. It is, of course, the very existence of these genuine differences that is the motivation behind the development of techniques for measurement directly from the rotor. The relatively small magnitude of the measured data compounded the difficulties encountered.

\section{Axial vibration measurement}

To measure the axial vibration set, aligning the laser beam so that it is parallel to the shaft rotation axis $\left(\beta=90^{\circ}\right)$, makes the measured velocity equal to: 


$$
\begin{aligned}
U_{m}= & {\left[V_{z}-\left(\dot{\theta}_{x}+\Omega \theta_{y}\right) a_{y}+\left(\dot{\theta}_{y}-\Omega \theta_{x}\right) a_{x}\right] } \\
& +y_{0}\left[\dot{\theta}_{x}+\Omega \theta_{y}\right]-x_{0}\left[\dot{\theta}_{y}-\Omega \theta_{x}\right]
\end{aligned}
$$

This shows that an axial vibration measurement is cross-sensitive to a combination of radial displacements, pitch and yaw. In equation (7), terms including the pitch and yaw sets of vibration terms are dependent on the offsets $x_{0}$ and $y_{0}$, and this is of significance, for example, in studies such as $[2,3,5]$ where the measurement point is offset from the shaft rotation axis. The sensitivity to these terms can be eliminated if it is acceptable to align the laser beam so that it is collinear with the shaft rotation axis, in which case $x_{0}=y_{0}=0$. In studies such as [6] where the laser beam is scanned, $\alpha, \beta, x_{0}$ and $y_{0}$ are all functions of time. Even if the variation in $\alpha$ and $\beta$ can be considered small, changes in $x_{0}$ and $y_{0}$ may still be significant. From an alternative perspective, the incident point on the scanning mirror can be taken as the reference point in which case $x_{0}$ and $y_{0}$ can be constant (possibly zero) but $z_{0}$ is no longer zero.

\section{Rotational vibration measurement}

While it is not possible to measure any of the three rotational vibration sets in equation (5) with a single laser beam, the Laser Torsional Vibrometer, a dual beam instrument, has been used successfully to measure the rotation speed set [13] and two such instruments have been used for initial assessment of pitch and yaw vibration [8]. Using equation (5) as the building block, the velocity measured by a combination of any number of beams can be formulated and this will be the subject of a further publication. 


\section{TOTAL VELOCITY MEASURED AT A FIXED POINT BY A CONTACTING TRANSDUCER}

To place the description of the velocity sensed by a laser vibrometer in its proper context, it is useful to compare equation (5) with its equivalent for a contacting transducer. For the purposes of the comparison, the "contacting transducer" is "attached" to non-rotating and then rotating structures. Of course the latter arrangement is fraught with practical difficulties but a comparison of the velocities sensed is useful in underlining the difficulty in making vibration measurements directly from rotors. The velocity measured by a contacting transducer fixed to a point on a vibrating structure can be derived in a similar way to the expression for the velocity sensed by a laser beam.

For the laser vibrometer, a combination of equations (1) and (2) gives the velocity of the measurement point $\mathrm{P}$ as:

$\vec{V}_{P}=\vec{V}_{0}+\left(\vec{\omega} \times \vec{r}_{0}\right)-(\vec{\omega} \times \vec{A})+(\vec{\omega} \times p \hat{b})$

The derivation of the velocity measured by a contacting transducer differs in that, unlike the incident point of the laser beam, the position of its measurement point Q remains fixed on the structure. However, as the structure tilts, the position of the measurement point deviates from its initial position in space by a small amount so that:

$$
\vec{V}_{Q}=\vec{V}_{0}+\left(\vec{\omega} \times\left[\vec{r}_{0}^{\prime}+\left(\vec{\theta} \times \vec{r}_{0}^{\prime}\right)\right]\right)=\vec{V}_{0}+\left(\vec{\omega} \times \vec{r}_{0}^{\prime}\right)+\vec{\omega} \times\left(\vec{\theta} \times \vec{r}_{0}^{\prime}\right)
$$


where $\vec{\theta}$ is the angular vibration displacement of the shaft element due to pitch, yaw and roll. $\vec{r}_{0}^{\prime}$ is comparable with $\vec{r}_{0}$ but differs from $\vec{r}_{0}$ in that it is a function of time and is used to account for the gross changes of the measurement point in space due to rotation of the shaft. This will be developed further in the second section of Appendix 1.

It is interesting to compare equations (8) and (9) which show two common terms, “ $\vec{V}_{o}+\left(\vec{\omega} \times \vec{r}_{0}\right)$," and additional terms which account for the change in the measurement point as the structure vibrates and rotates.

In equation (8) the first of the additional terms, $(\vec{\omega} \times \vec{A})$, accounts for the effect of the change in the point of incidence of the laser beam on the target due to translation of the structure. The second term, $(\vec{\omega} \times p \hat{b})$, accounts for the changes in space of the point of incidence due to target motion and/or shape but this component is not present in the measured velocity because it is always perpendicular to the incident laser beam. The additional term in equation (9), $\vec{\omega} \times\left(\vec{\theta} \times \vec{r}_{0}^{\prime}\right)$, accounts for the effect of changes in the measurement point as the structure tilts. In addition to these differences, a further effect in the measured velocity results from changes in the sensitivity vector for the contacting transducer. While the non-contacting transducer measures $\left(\hat{b} \cdot \vec{V}_{P}\right)$, where $\hat{b}$ is usually a constant, the contacting transducer measures $\left(\hat{e} \cdot \vec{V}_{Q}\right)$, where:

$\hat{e}=\hat{e}_{0}+\left(\vec{\theta} \times \hat{e}_{0}\right)$

This accounts for the small deviation of the sensitivity vector from its undeflected direction, $\hat{e}_{0}$, as the structure tilts. 
Section 2 of Appendix 1 details the derivation of the velocity measured by a contacting transducer fixed to point $\mathrm{Q}$ on a structure undergoing an arbitrary vibration, including rotation about its spin axis. In what follows, a comparison is made between the velocity measured by a contacting transducer and the velocity measured by a laser vibrometer.

\section{COMPARISON OF NON-CONTACTING AND CONTACTING TRANSDUCER OUTPUTS}

\subsection{NON-ROTATING STRUCTURES}

Figure 7 shows a typical measurement situation. The $y$ vibration velocity of a cantilever beam is to be measured at some position along its length. The axial locations of the measurement point and the co-ordinate axes are chosen to coincide, with the axis of the cantilever beam defining the direction of the $z$-axis.

For a contacting transducer with its base fixed to point $\mathrm{Q}$ and its sensitivity axis orientated initially in the positive $y$ direction, from equations (A1.7) and (A1.10) with $\Omega=0$, the velocity measured is:

$$
\begin{aligned}
U_{Q} & =V_{y}+\dot{\theta}_{z}\left(x_{0}-\theta_{z} y_{0}\right)-\dot{\theta}_{x}\left(\theta_{x} y_{0}-\theta_{y} x_{0}\right) \\
& +\theta_{x}\left[V_{z}+\dot{\theta}_{x}\left(y_{0}+\theta_{z} x_{0}\right)-\dot{\theta}_{y}\left(x_{0}+\theta_{z} y_{0}\right)\right] \\
& -\theta_{z}\left[V_{x}+\dot{\theta}_{y}\left(\theta_{x} y_{0}-\theta_{y} x_{0}\right)-\dot{\theta}_{z}\left(y_{0}+\theta_{z} x_{0}\right)\right]
\end{aligned}
$$

To make the same intended measurement with a laser vibrometer requires the beam to be incident on a point $\mathrm{P}$, where the points $\mathrm{P}$ and $\mathrm{Q}$ are initially coincident, and aligned parallel to the $y$ axis making $\alpha=90^{\circ}, \beta=0^{\circ}, z_{0}=0$. From equation (5) the measured velocity is: 


$$
U_{m}=V_{y}+\dot{\theta}_{z}\left(x_{0}-a_{x}\right)+\dot{\theta}_{x} a_{z}
$$

Equations (11a\&b) show that, neglecting the higher order terms, the two measurements are

both equal to $\left(V_{y}+\dot{\theta}_{z} x_{0}\right)$, which is readily accepted as the $y$ velocity of the measurement point. The additional terms in equation (11a) are due to the change in direction of the transducer sensitivity axis as the structure tilts (those enclosed in square brackets) and to the change in position of the measurement point in space as the structure tilts. The additional terms in equation (11b) are due to the measurement point changing position on the target, while remaining essentially fixed in space because changes due to target shape are unimportant.

\subsection{ROTATING STRUCTURES}

On a rotating structure the time dependence of $\hat{e}$ would make for a very complicated description of the measured velocity, but one that can be given by expansion of equation (A1.6) using equations (A1.7), modified as described in section 2 of Appendix 1, and (A1.10). If it could be achieved, an interesting contacting transducer measurement would be one in which the sensitivity direction remains fixed despite the target motion. It is interesting to compare the output of this 'ideal' contacting transducer with the fixed direction laser vibrometer measurement.

For a measurement of radial vibration in the $y$ direction the velocity measured by the 'ideal' contacting transducer is the velocity component in the $y$ direction. Using equation (A1.10) and letting the axial location of the transducer define the location of the co-ordinate axes:

$$
\left(\hat{y} \cdot \vec{V}_{Q}\right)=V_{y}-\left(\dot{\theta}_{x}+\Omega \theta_{y}\right)\left[\theta_{x} G(t)-\theta_{y} F(t)\right]+\left(\dot{\theta}_{z}+\Omega\right)\left[F(t)-\theta_{z} G(t)\right]
$$


where $F(t)=x_{0} \cos \Omega t-y_{0} \sin \Omega t \quad$ and $\quad G(t)=x_{0} \sin \Omega t+y_{0} \cos \Omega t$. Making the same intended measurement using a laser vibrometer, equation (5) would give:

$U_{m}=V_{y}+\left(\dot{\theta}_{x}+\Omega \theta_{y}\right)\left[a_{z}\right]+\left(\dot{\theta}_{z}+\Omega\right)\left[x_{0}-a_{x}\right]$

Unlike equations (11a\&b), after neglecting the higher order terms, equations (12a\&b) differ in their cross-sensitivities to rotation speed and roll. The terms enclosed in the square brackets in both equations account for the change in position of the measurement point. The differences between these sets of terms occur because the positions of the measurement points change in different ways. For the contacting transducer, displacement of the structure causes the measurement position to change position in space but remain in the same position relative to a rotating reference frame attached to the structure. In contrast, for the laser vibrometer, displacement of the structure causes the measurement point to change position on the target but it remains somewhere along a fixed line in space. However, this change in position along the line of the laser beam has no effect on the measured velocity as equation (4) shows. Thus, for the laser vibrometer, the extra terms are principally due to the change in position of the measurement point relative to a reference frame attached to the structure but not changing position in space.

The cross-sensitivity of the measurements can be compared for both transducers by studying the dominant 'error velocity' term, $\Omega F(t)$ for the contacting transducer or $\Omega a_{x}$ for the laser vibrometer. Considering a harmonic vibration at frequency $\omega_{v}$ for the purposes of comparison, these terms can be written as: 
$\Omega F(t)=\Omega \sqrt{x_{0}^{2}+y_{0}^{2}} \cos (\Omega t+\phi)$

$\Omega a_{x}=\Omega A_{x} \cos \left(\omega_{v} t+\phi^{\prime}\right)$

where $\phi$ and $\phi^{\prime}$ are phase terms and $A_{x}$ is the amplitude of the vibration displacement in the $x$ direction i.e. perpendicular to that of the intended measurement.

As $\sqrt{x_{0}^{2}+y_{0}^{2}}$, which for a cylindrical shaft is equal to the shaft radius, is generally much larger than displacement amplitudes, the cross-sensitivity is much greater even for this 'ideal' contacting transducer. However, as $x_{0}, y_{0}, \phi$ and $\Omega$ are known or can be measured, the potential to subtract this component from the contacting transducer's measured velocity does exist while this is not possible for the laser vibrometer as $A_{x}$ and $\phi^{\prime}$ are unknown - indeed, they are part of the required measurement. Unfortunately, because it is likely that $\Omega F(t)>>V_{y}$, this subtraction would be very prone to error.

The cross-sensitivity in the laser vibrometer measurements, which can mask the intended measurement entirely, can be overcome for all but synchronous vibrations by post processing [12]. The cross-sensitivity problems in the laser vibrometer measurement are dependent on the vibration displacement frequencies whereas the cross-sensitivity in the contacting transducer measurement would principally only occur at synchronous frequencies. However, any torsional vibrations or speed fluctuations would cause time dependence in $\Omega$ and introduce cross-sensitivity into the contacting transducer measurements at additional frequencies.

These comparisons emphasise the difficulties encountered in attempting to measure vibrations directly from a rotating target not just for non-contacting transducers, as documented previously, but also for contacting transducers. The potential of laser vibrometry for such 
challenging applications is not so much diminished by the issues raised in this paper as it is emphasised by the problems that would be encountered even if a suitable contacting transducer could be devised.

\section{CONCLUSIONS}

This paper has developed a comprehensive theory to describe the velocity sensed by a single laser beam incident on a rotating structure requiring three translational and three rotational co-ordinates to describe its vibratory motion fully. The theory is equally applicable to measurements on targets with simpler motions, such as non-rotating targets, allowing the vibration engineer to determine the vibration component sensitivity of any measurement with any laser beam orientation.

Arbitrary motion of any arbitrarily shaped component can cause the point of incidence of the laser beam on the target surface to change. This feature of such measurements has been incorporated in the new theory and insensitivity to target shape proved in the most general fashion.

Six separate vibration sets, each a combination of motion parameters, appear in the full expression for vibration velocity sensitivity. The $x$ radial, $y$ radial, and axial sets can be isolated by appropriate geometric set-up of the laser beam while the pitch and yaw vibration sets and the rotation speed set cannot be isolated with a single laser beam. Resolution of individual motion components within each set has been shown not to be possible by any geometric arrangement of the laser beam or by introduction of additional laser beams.

Many previous analyses have predicted satisfactory and straightforward application because only single vibration components have been considered. For a rotating shaft undergoing arbitrary motion, direct measurement of radial or axial vibration is not possible because the 
measurement will always be sensitive to other motion components. Approximate solutions to resolve steady-state, non-synchronous, radial vibrations are under development based on postprocessing of simultaneous measurements [7,12].

Examples of how the new theory can be applied have been presented and have shown agreement with the specific cases considered in previous work, underlining the theory's versatility in predicting laser vibrometer sensitivity to target motion with six degrees-offreedom. The model of velocity sensitivity in a laser vibrometer measurement has been put into context by comparison with the velocity sensitivity of a contacting transducer under equivalent conditions. The comparison serves to emphasise the challenging nature of measurement directly from rotating components and, even for an 'ideal' contacting transducer able to maintain its sensitivity axis during rotation, important cross-sensitivities would exist. The comprehensive nature of the model presented will enable laser vibrometer measurements directly from rotors to be interpreted correctly and attention can now be turned to postprocessing instrument outputs to give the best quality data possible. The general nature of the theory means that it can be easily extended to cover measurements made with multiple beams for rotational vibration measurements and this will be the subject of a further publication. Extension of the theory to analysis of axially flexible rotating discs is the subject of continuing work. 


\section{APPENDIX 1}

\section{A1 VELOCITY MEASURED BY AN INCIDENT LASER BEAM}

The origin of the translating reference frame $x y z$ is fixed to a point on the centre line of the shaft with the undeflected shaft rotation axis defining the direction and position of the $z$-axis. The configuration is depicted in Figure 1 with the time dependent unit vector $\hat{z}_{R}$ defining the changing direction of the shaft rotation axis, which deviates from the $z$-axis as the shaft tilts. The velocity measured by the laser vibrometer, $U_{m}$, is the component of the velocity of the point of incidence in the direction of the incident beam.

The velocity of the point of incidence $P$, given by equation (1), can be expanded as:

$$
\begin{aligned}
\vec{V}_{P} & =\left(V_{x} \hat{x}+V_{y} \hat{y}+V_{z} \hat{z}\right)+\dot{\theta}_{x}\left(\hat{x} \times \vec{r}_{p}\right) \\
& +\dot{\theta}_{y}\left(\hat{y} \times \vec{r}_{p}\right)+\dot{\theta}_{z}\left(\hat{z} \times \vec{r}_{p}\right)+\Omega\left(\hat{z}_{R} \times \vec{r}_{p}\right)
\end{aligned}
$$

where $V_{x}, V_{y}$ and $V_{z}$ are the translational vibration velocities of the origin $\mathrm{O}$ in the $x, y$ and $z$ directions, $\Omega$ is the total rotation speed of the axial shaft element (combining shaft rotation speed and any torsional vibration of the axial element) and $\dot{\theta}_{x}, \dot{\theta}_{y}$ and $\dot{\theta}_{z}$ are the angular vibration velocities of the shaft around the $x, y$ and $z$ axes, referred to as pitch, yaw and roll respectively.

The small angular deviation of $\hat{z}_{R}$ from $\hat{z}$ can be related to the shaft pitch and yaw with $\hat{z}_{R}$ written in terms of a constant component, $\hat{z}$, and a time dependent component:

$$
\hat{z}_{R}=\hat{z}+(\vec{\theta} \times \hat{z})=\hat{z}+\theta_{y} \hat{x}-\theta_{x} \hat{y}
$$


where $\vec{\theta}$ is the angular vibration displacement of the shaft element due to pitch, yaw and roll. This eliminates the need for the time dependent unit vector $\hat{z}_{R}$ and the velocity of the point $\mathrm{P}$ can now be written only in terms of the three orthogonal vectors $\hat{x}, \hat{y}$ and $\hat{z}$ :

$$
\begin{aligned}
\vec{V}_{P} & =\left(V_{x} \hat{x}+V_{y} \hat{y}+V_{z} \hat{z}\right)+\left(\dot{\theta}_{x}+\Omega \theta_{y}\right)\left(\hat{x} \times \vec{r}_{P}\right) \\
& +\left(\dot{\theta}_{y}-\Omega \theta_{x}\right)\left(\hat{y} \times \vec{r}_{P}\right)+\left(\dot{\theta}_{z}+\Omega\right)\left(\hat{z} \times \vec{r}_{P}\right)
\end{aligned}
$$

Substituting the position of the incident point, $\vec{r}_{P}$, given by equation (2), into equation (A1.3) and using equation (4), the velocity measured by a laser vibrometer incident on a rotating shaft may be written as:

$$
\begin{aligned}
U_{m} & =\hat{b} \cdot\left(V_{x} \hat{x}+V_{y} \hat{y}+V_{z} \hat{z}\right)+\left(\dot{\theta}_{x}+\Omega \theta_{y}\right) \hat{b} \cdot\left(-\left(z_{0}-a_{z}\right) \hat{y}+\left(y_{0}-a_{y}\right) \hat{z}\right) \\
& +\left(\dot{\theta}_{y}-\Omega \theta_{x}\right) \hat{b} \cdot\left(\left(z_{0}-a_{z}\right) \hat{x}-\left(x_{0}-a_{x}\right) \hat{z}\right) \\
& +\left(\dot{\theta}_{z}+\Omega\right) \hat{b} \cdot\left(-\left(y_{0}-a_{y}\right) \hat{x}+\left(x_{0}-a_{x}\right) \hat{y}\right)
\end{aligned}
$$

where $a_{x}, a_{y}$ and $a_{z}$ are the translational vibration displacements of the origin $\mathrm{O}$ in the $x, y$ and $z$ directions and $x_{0}, y_{0}$ and $z_{0}$ are the co-ordinates (measured from the undeflected axes as shown in figure 3) of the known point on the line of the beam.

In order to make this equation of more direct practical use, $\hat{b}$ needs to be described in terms of measurable parameters. Figure 4 shows how $\hat{b}$ can be described as a combination of two angles; with $\hat{b}=\hat{x}$ initially, rotating first by an angle $\beta$ around $\hat{y}$, then by an angle $\alpha$ around $\hat{z}$. These two rotations are finite and, therefore, this order of rotation must be maintained. In Cartesian form $\hat{b}$ is given by: 
$\hat{b}=\cos \beta \cos \alpha \hat{x}+\cos \beta \sin \alpha \hat{y}-\sin \beta \hat{z}$

Substituting equation (A1.5) into equation (A1.4) gives the full expression for the velocity measured by a laser vibrometer, orientated according to the angles $\alpha$ and $\beta$ and incident on a rotating shaft, as equation (5) in the main paper.

\section{A2 VELOCITY MEASURED BY A CONTACTING TRANSDUCER}

The velocity, $\vec{U}_{Q}$, measured at a point $\mathrm{Q}$ to which the transducer is fixed is the component of the velocity of $\mathrm{Q}$ in the direction of the transducer's sensitivity axis, defined by the unit vector $\hat{e}$, such that:

$\vec{U}_{Q}=\hat{e} \cdot \vec{V}_{Q}$

As the component tilts, however, the direction of the sensitivity axis deviates a small amount from its initial direction, $\hat{e}_{0}$, such that:

$\hat{e}=\hat{e}_{0}+\left(\vec{\theta} \times \hat{e}_{0}\right)=\left(e_{x}+\theta_{y} e_{z}-\theta_{z} e_{y}\right) \hat{x}+\left(e_{y}+\theta_{z} e_{x}-\theta_{x} e_{z}\right) \hat{y}+\left(e_{z}+\theta_{x} e_{y}-\theta_{y} e_{x}\right) \hat{z}$

where

$\hat{e}_{0}=e_{x} \hat{x}+e_{y} \hat{y}+e_{z} \hat{z}$

For a non-vibrating structure, rotating about its spin axis, defined by the unit vector $\hat{z}$, the instantaneous position of a point $\mathrm{Q}$ on the structure, $\vec{r}_{0}^{\prime}$, can be written as: 
$\vec{r}_{0}^{\prime}=F(t) \hat{x}+G(t) \hat{y}+z_{0} \hat{z}$

where $F(t)=x_{0} \cos \Omega t-y_{0} \sin \Omega t$ and $G(t)=x_{0} \sin \Omega t+y_{0} \cos \Omega t$.

As a result of angular vibration of the structure the position vector becomes:

$\vec{r}_{Q}=\vec{r}_{0}^{\prime}+\left(\vec{\theta} \times \vec{r}_{0}^{\prime}\right)=F(t) \hat{x}_{R}+G(t) \hat{y}_{R}+z_{0} \hat{z}_{R}$

where $x_{0}, y_{0}$ and $z_{0}$ define the initial position of the point in the translating reference frame $x y z, \Omega$ is the angular velocity of the shaft about the spin axis and $\hat{x}_{R}, \hat{y}_{R}$ and $\hat{z}_{R}$ are the unit vectors defining a translating and tilting, but non-rotating, reference frame fixed to the component.

As previously described by equation (A1.2), the small angular deviations of $\hat{x}_{R}, \hat{y}_{R}$ and $\hat{z}_{R}$ from their undeflected positions, $\hat{x}, \hat{y}$ and $\hat{z}$, can be related to the pitch, yaw and roll of the component such that the instantaneous position of a point $\mathrm{Q}, \vec{r}_{Q}$, on the component can be written as:

$\vec{r}_{Q}=\left[F(t)+z_{0} \theta_{y}-\theta_{z} G(t)\right] \hat{x}+\left[G(t)-z_{0} \theta_{x}+\theta_{z} F(t)\right] \hat{y}+\left[z_{0}+\theta_{x} G(t)-\theta_{y} F(t)\right] \hat{z}$

By inserting this expression for the position of point Q into equation (A1.3), which describes the velocity of any point on a rotating component, the velocity of the point $\mathrm{Q}, \vec{V}_{Q}$, can be written as: 


$$
\begin{aligned}
\vec{V}_{Q} & =\left(V_{x} \hat{x}+V_{y} \hat{y}+V_{z} \hat{z}\right)+\left(\dot{\theta}_{x}+\Omega \theta_{y}\right)\left(\left[G(t)-z_{0} \theta_{x}+\theta_{z} F(t)\right] \hat{z}-\left[z_{0}+\theta_{x} G(t)-\theta_{y} F(t)\right] \hat{y}\right) \\
& +\left(\dot{\theta}_{y}-\Omega \theta_{x}\right)\left(\left[z_{0}+\theta_{x} G(t)-\theta_{y} F(t)\right] \hat{x}-\left[F(t)+z_{0} \theta_{y}-\theta_{z} G(t)\right] \hat{z}\right) \\
& +\left(\dot{\theta}_{z}+\Omega\right)\left(\left[F(t)+z_{0} \theta_{y}-\theta_{z} G(t)\right] \hat{y}-\left[G(t)-z_{0} \theta_{x}+\theta_{z} F(t)\right] \hat{x}\right)
\end{aligned}
$$

Combination of this equation with equations (A1.7a\&b), with equation (A1.7b) modified to include the gross rotation of the transducer in a similar fashion to equation (A1.8a), is straightforward and will reveal the velocity measured by the contacting transducer fixed to a rotating structure undergoing arbitrary motion. Although this is not done here for brevity, equations (A1.7) and (A1.10) will prove useful in the comparison of non-contacting and contacting transducers in section 4 of the main paper.

\section{ACKNOWLEDGEMENTS}

J.R. Bell wishes to acknowledge the continued support of the Engineering and Physical Sciences Research Council. The authors also acknowledge the comments on the manuscript made by Professor Neil Halliwell. 


\section{REFERENCES}

1. Q.V. DAVIS and W.K. KULCZYK 1969 Nature 222, 475-476. Vibrations of turbine blades measured by means of a laser.

2. T.A. RIENER, A.C. GODING and F.E. TALKE 1988 IEEE Transactions on Magnetics 24(6), 2745-2747. Measurement of head/disc spacing modulation using a two channel fiber optic laser Doppler vibrometer.

3. R.W. WLEZEIN, D.K. MIU and V. KIBENS 1984 Optical Engineering 24(4), 436442. Characterization of rotating flexible disks using a laser Doppler vibrometer.

4. R.A. COOKSON and P. BANDYOPADHYAY 1980 Transactions of the ASME Journal of Engineering for Power 102, 607-612. A fiber-optics laser Doppler probe for vibration analysis of rotating machines.

5. A.K. REINHARDT, J.R. KADAMBI and R.D. QUINN 1995 Transactions of the ASME Journal of Engineering for Gas Turbines and Power 117, 484-488. Laser vibrometry measurements on rotating blade vibrations.

6. A.B. STANBRIDGE and D.J. EWINS 1995 Proceedings of ASME Design Engineering Technical Conference, Boston, USA 3(B), 1207-1213. Modal testing of rotating discs using a scanning LDV.

7. S.J. ROTHBERG and N.A. HALLIWELL 1994 Transactions of the ASME Journal of Vibration and Acoustics 116(3), 326-331. Vibration measurements on rotating machinery using laser Doppler velocimetry.

8. T.J. MILES, M. LUCAS, N.A. HALLIWELL and S.J. ROTHBERG 1999 Journal of Sound and Vibration 226(3), 441-467. Torsional and bending vibration measurement on rotors using laser technology. 
9. J.F. VIGNOLA and B.H. HOUSTON 1993 ASME Winter Annual Meeting, New Orleans, Louisiana, USA ISSN 0402-1215, 1-10. The design of a three dimensional laser vibrometer.

10. E.H. BOKELBERG, H.J. SOMMER and M.W. TRETHEWEY 1994 Journal of Sound and Vibration 178(5), 643-654. A six degree-of-freedom laser vibrometer, part I: theoretical development.

11. S.J. ROTHBERG and N.A. HALLIWELL 1992 Proceedings of IMechE - $5^{\text {th }}$ International Conference on Vibrations in Rotating Machinery, Bath, UK 409-415. On the use of laser vibrometry for rotating machinery measurements.

12. J.R. BELL and S.J. ROTHBERG 1998 Proceedings of SPIE - 3rd International Conference on Vibration Measurements by Laser Techniques: Advances and Applications, Ancona, Italy 3411, 14-22. Radial vibration measurements on rotors using laser vibrometry: a first practical solution to the cross-sensitivity problem.

13. N.A. HALLIWELL, C.J.D. PICKERING and P.G. EASTWOOD 1984 Journal of Sound and Vibration 93(4), 588-592. The laser torsional vibrometer: a new instrument. 
LASER VIBROMETERS AND CONTACTING TRANSDUCERS, TARGET ROTATION AND 6 DEGREE-OF-FREEDOM VIBRATION: WHAT DO WE REALLY MEASURE?

\author{
J.R. BELL and S.J. ROTHBERG
}

\title{
FIGURES
}

Figure 1. Definition of axes and of the point $\mathrm{P}$ on a vibrating and rotating structure.

Figure 2. Changes in the point of incidence due to:

a. Translation.

b. Rotation.

c. Pitch and Yaw. (Tilt).

Figure 3. Change in position vector caused by target motion and arbitrary shape.

Figure 4. Orientation of laser beam.

Figure 5. Test rig for validation of terms combining axial and angular motions.

Figure 6. Experimental validation of error terms in radial vibration measurements.

a. $\Omega \theta_{x} a_{z}$.

b. $\dot{\theta}_{y} a_{z}$.

Figure 7. Vibration measurement on a cantilever beam. 


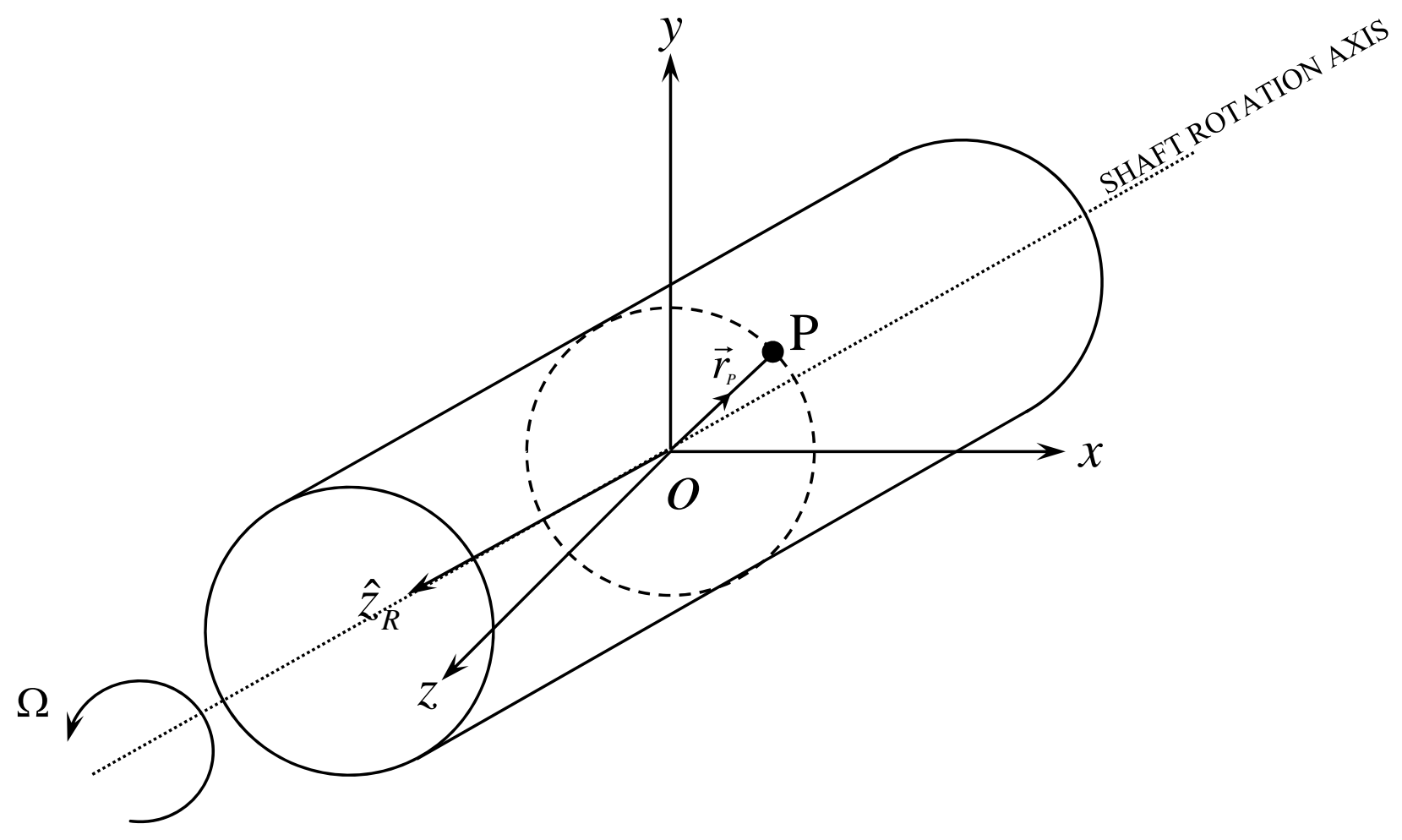

Figure 1

Laser Vibrometers and Contacting Transducers, Target Rotation and 6 Degree-of-Freedom Vibration: What do we really measure?

J.R. Bell and S.J. Rothberg

Loughborough University 


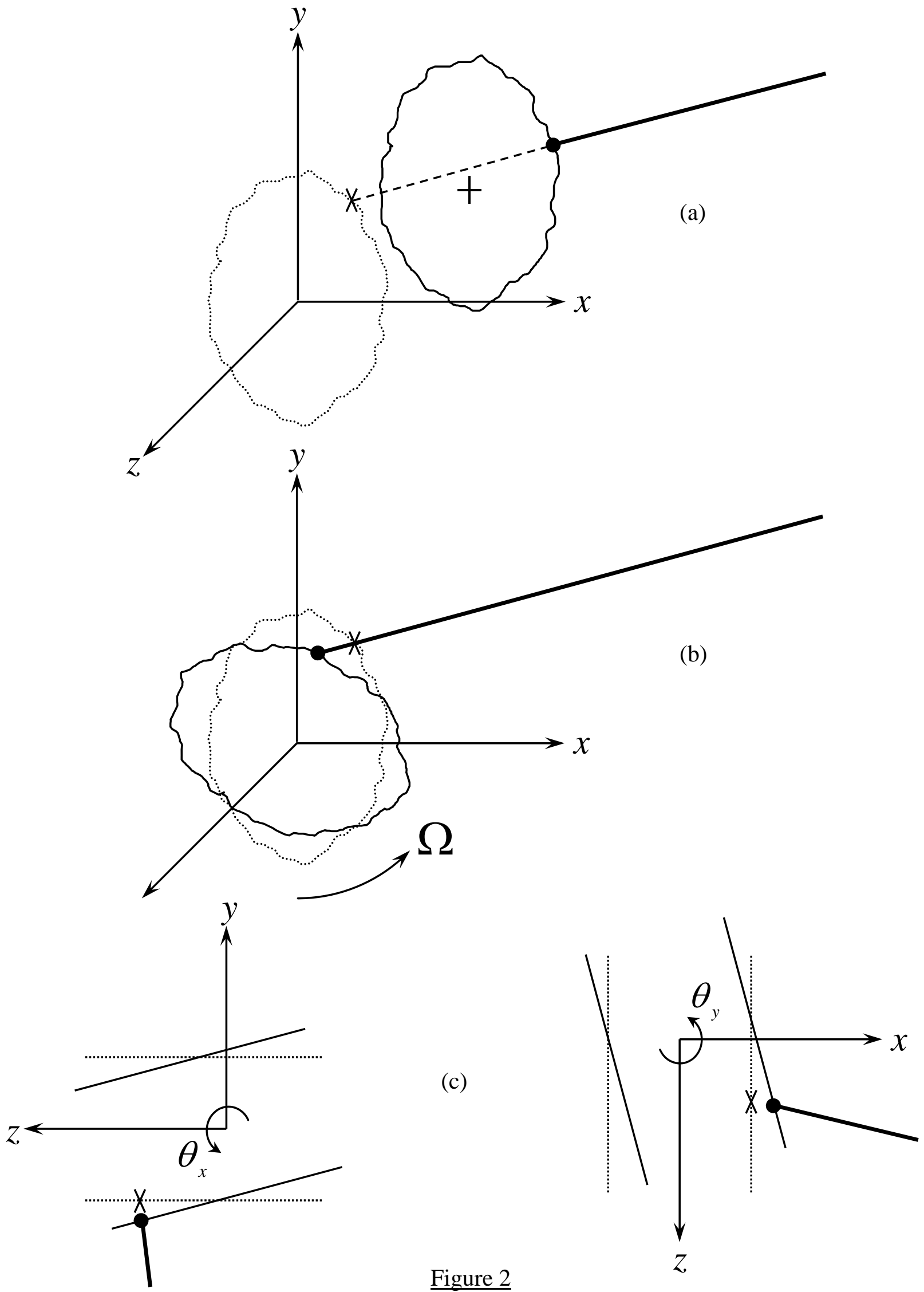

Laser Vibrometers and Contacting Transducers, Target Rotation and 6 Degree-of-Freedom Vibration: What do we really measure?

J.R. Bell and S.J. Rothberg Loughborough University 


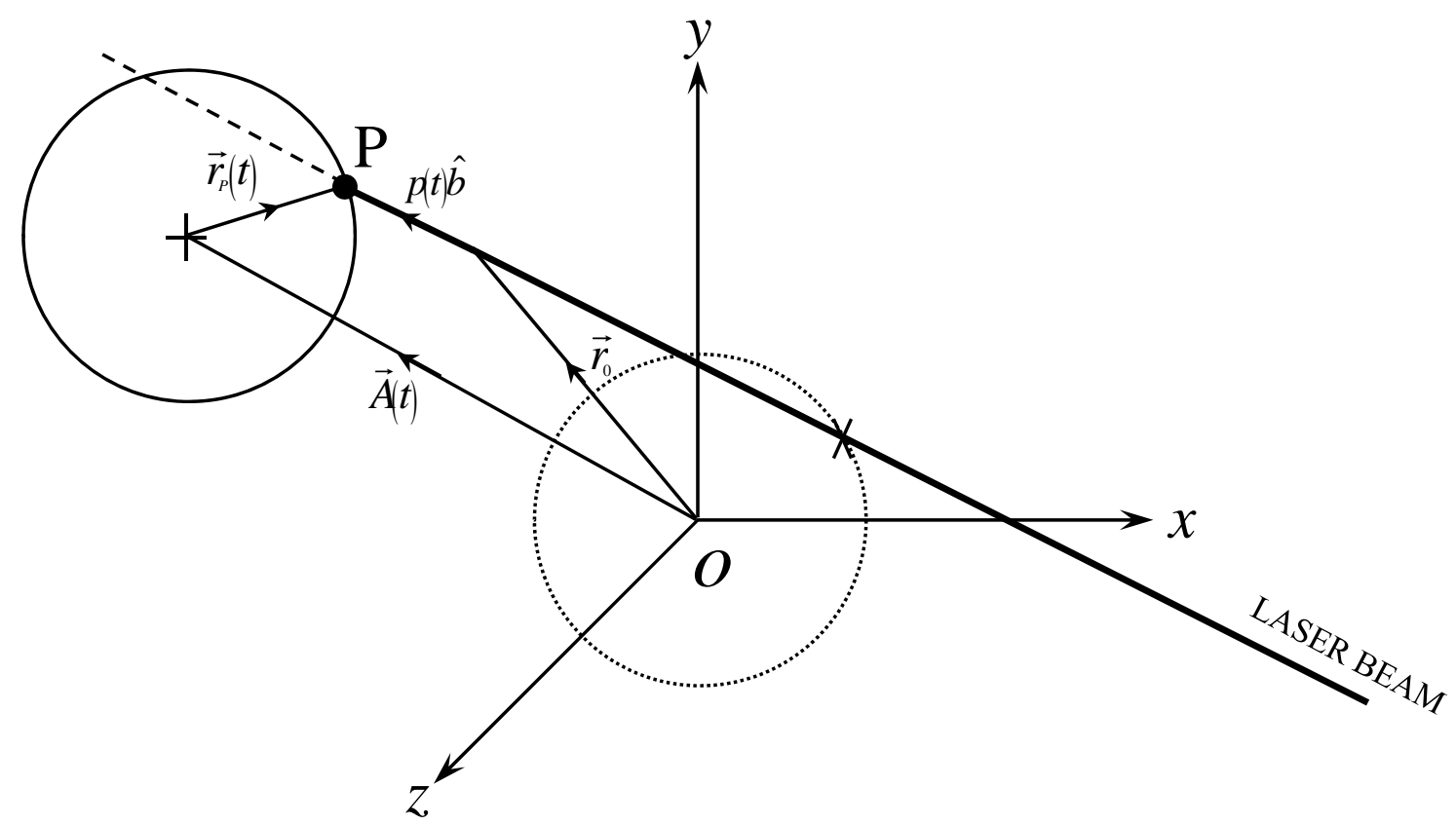

Figure 3

Laser Vibrometers and Contacting Transducers, Target Rotation and 6 Degree-of-Freedom Vibration: What do we really measure?

J.R. Bell and S.J. Rothberg

Loughborough University 


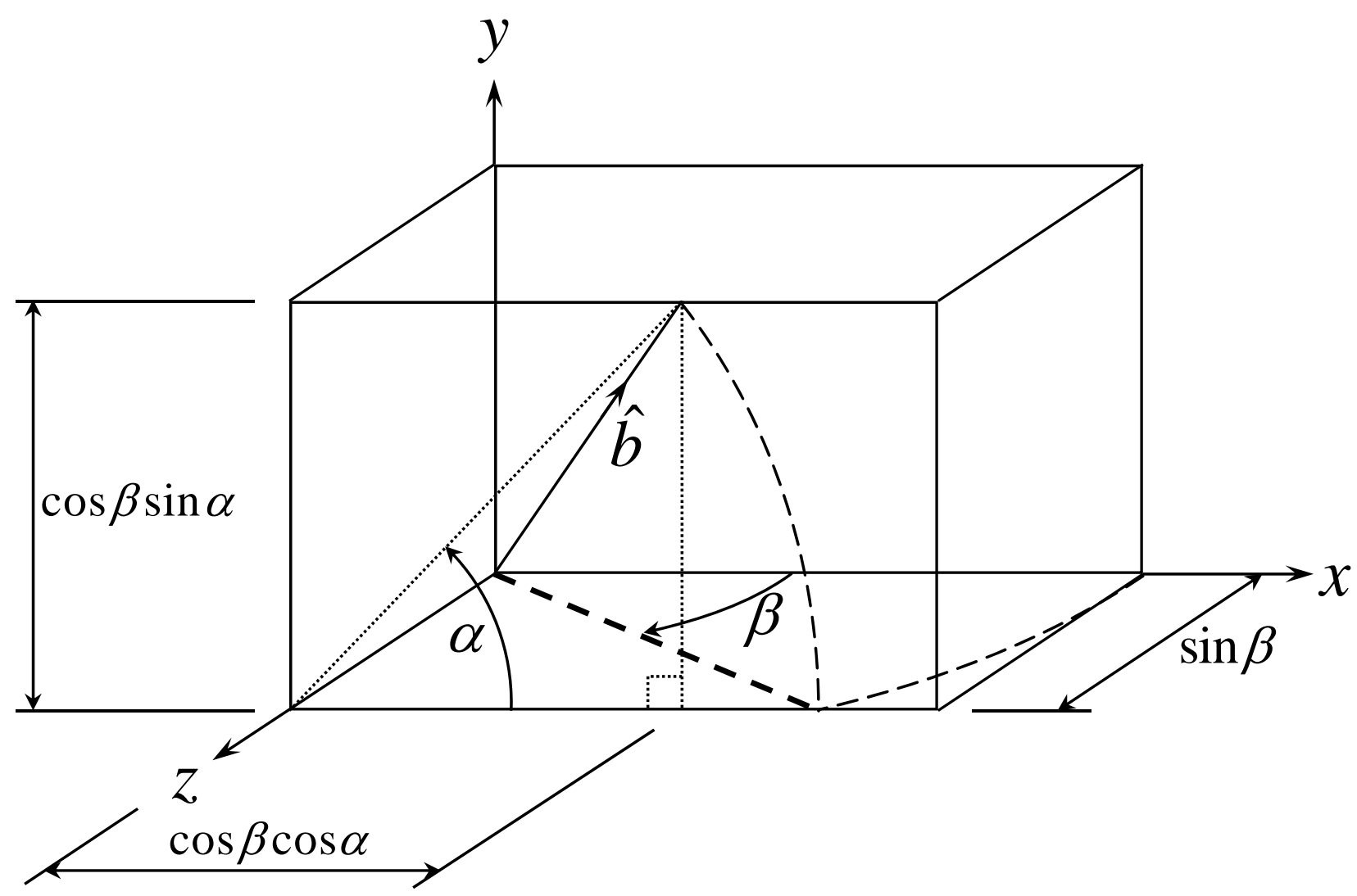

Figure 4

Laser Vibrometers and Contacting Transducers, Target Rotation and 6 Degree-of-Freedom Vibration: What do we really measure?

J.R. Bell and S.J. Rothberg

Loughborough University 


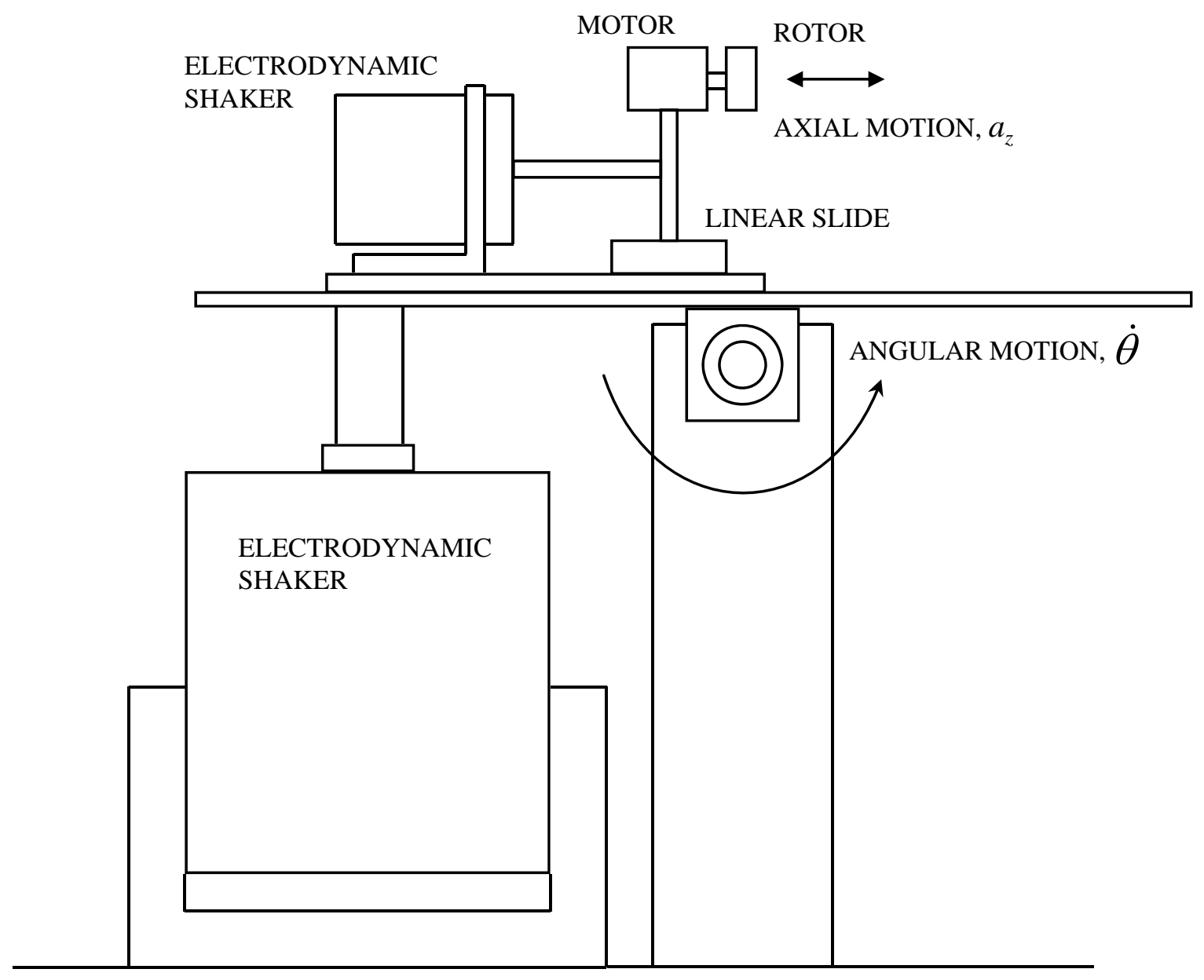

Figure 5

Laser Vibrometers and Contacting Transducers, Target Rotation and 6 Degree-of-Freedom Vibration: What do we really measure?

J.R. Bell and S.J. Rothberg

Loughborough University 


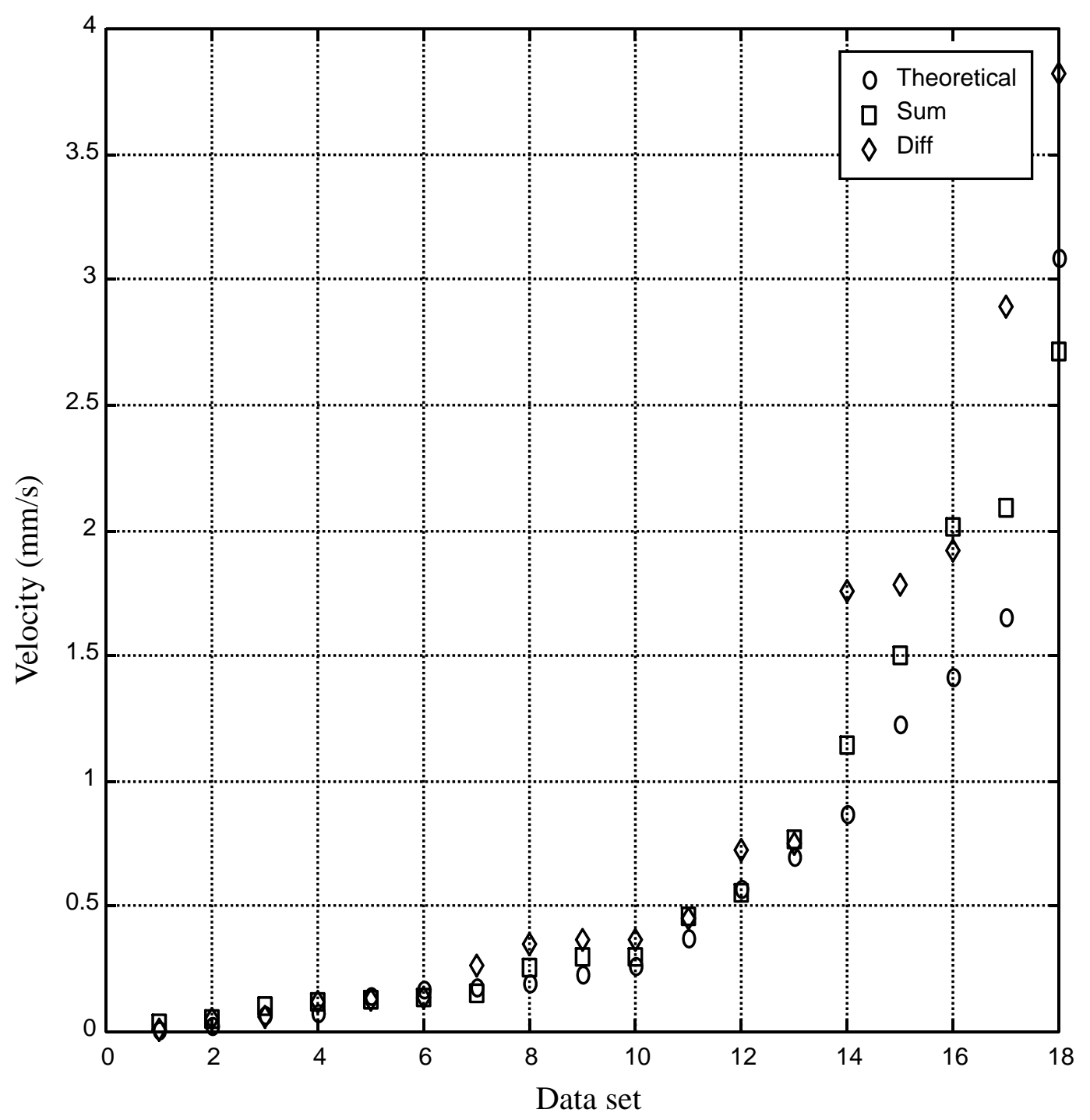

Figure 6a

Laser Vibrometers and Contacting Transducers, Target Rotation and 6 Degree-of-Freedom Vibration: What do we really measure?

J.R. Bell and S.J. Rothberg

Loughborough University 


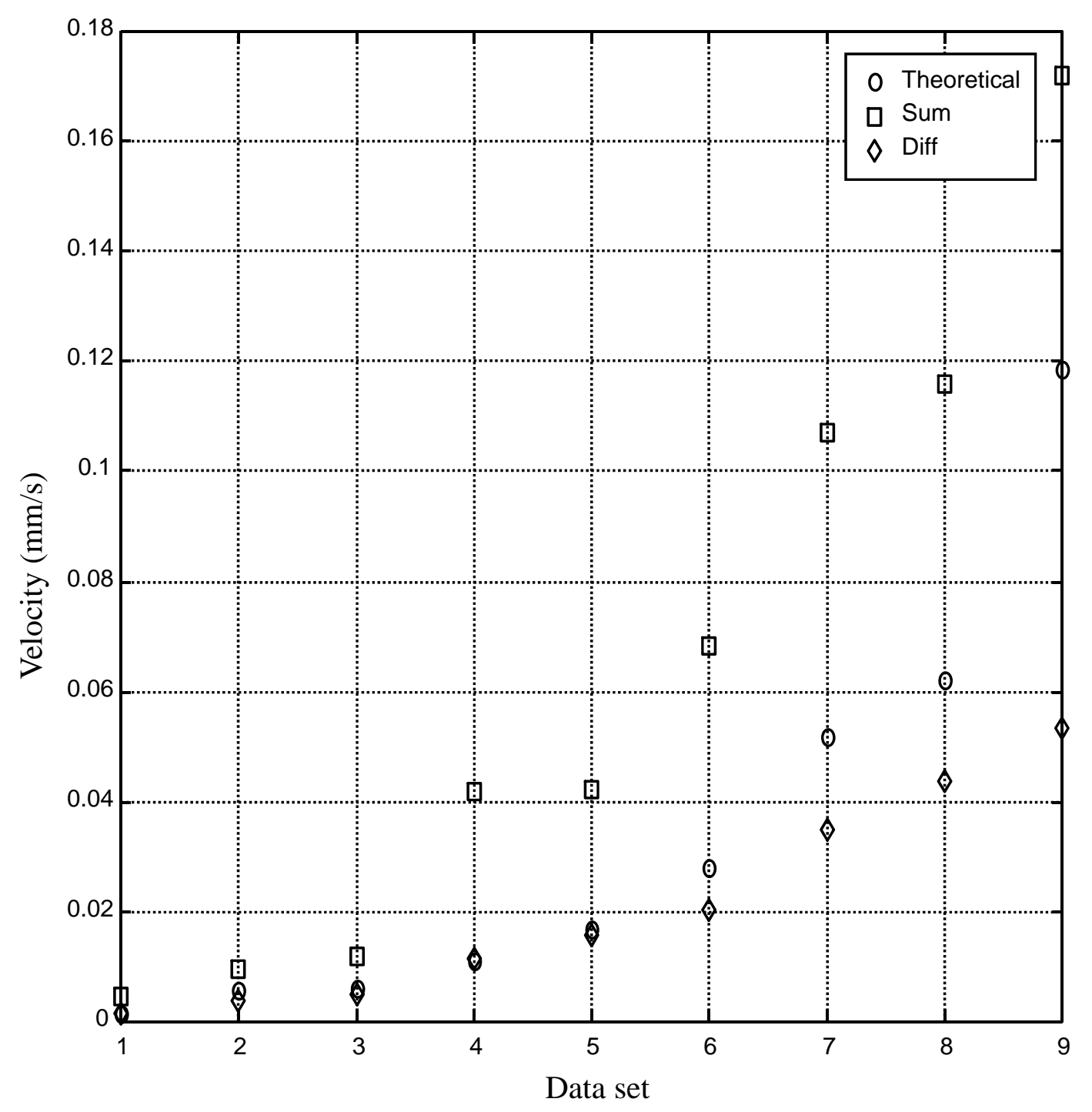

Figure 6b

Laser Vibrometers and Contacting Transducers, Target Rotation and 6 Degree-of-Freedom Vibration: What do we really measure?

J.R. Bell and S.J. Rothberg

Loughborough University 


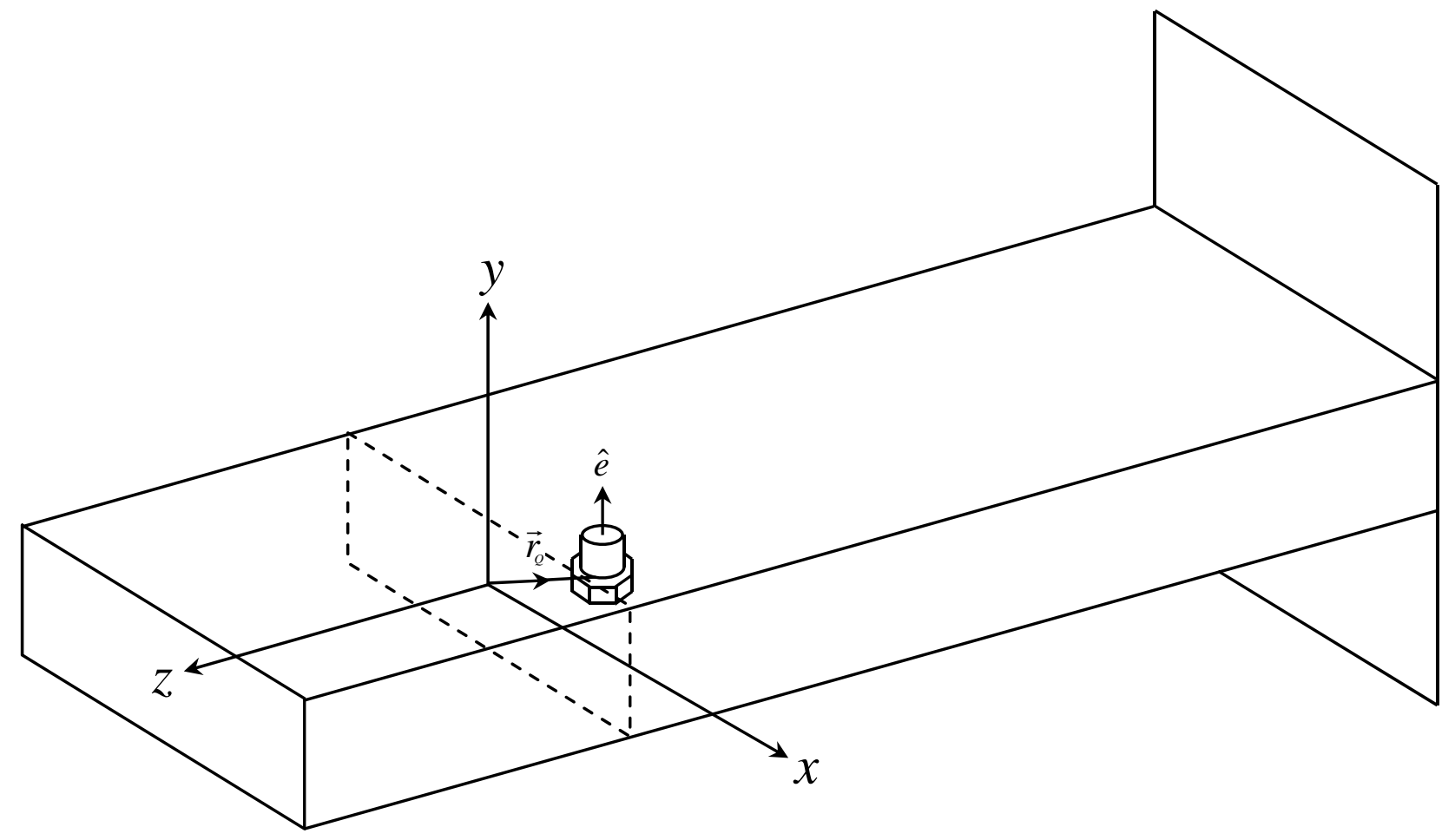

Figure 7

Laser Vibrometers and Contacting Transducers, Target Rotation and 6 Degree-of-Freedom Vibration: What do we really measure?

J.R. Bell and S.J. Rothberg

Loughborough University 